\title{
Effects of selenium on the survival and invasion of trophoblasts
}

\author{
Jee Yoon $\mathrm{Na}^{1}$, Jin Seok², Sohae Park², Jung Seok Kim', Gi Jin Kim² \\ 'Cheongshim International Academy, Gapyeong; '2Department of Biomedical Science, CHA University, Seongnam, Korea
}

Objective: Placental oxidative stress is known to be a factor that contributes to pregnancy failure. The aim of this study was to determine whether selenium could induce antioxidant gene expression and regulate invasive activity and mitochondrial activity in trophoblasts, which are a major cell type of the placenta.

Methods: To understand the effects of selenium on trophoblast cells exposed to hypoxia, the viability and invasive activity of trophoblasts were analyzed. The expression of antioxidant enzymes was assessed by reverse-transcription polymerase chain reaction. In addition, the effects of selenium treatment on mitochondrial activity were evaluated in terms of adenosine triphosphate production, mitochondrial membrane potential, and reactive oxygen species levels.

Results: Selenium showed positive effects on the viability and migration activity of trophoblast cells when exposed to hypoxia. Interestingly, the increased heme oxygenase 1 expression under hypoxic conditions was decreased by selenium treatment, whereas superoxide dismutase expression was increased in trophoblast cells by selenium treatment for 72 hours, regardless of hypoxia. Selenium-treated trophoblast cells showed increased mitochondrial membrane potential and decreased reactive oxygen species levels under hypoxic conditions for 72 hours.

Conclusion: These results will be used as basic data for understanding the mechanism of how trophoblast cells respond to oxidative stress and how selenium promotes the upregulation of related genes and improves the survival rate and invasive ability of trophoblasts through regulating mitochondrial activity. These results suggest that selenium may be used in reproductive medicine for purposes including infertility treatment.

Keywords: Antioxidants; Hypoxia; Invasiveness; Mitochondria; Reactive oxygen species; Selenium; Trophoblasts

\section{Introduction}

Trophoblasts form the outer layer of blastocysts, and contribute to diverse factors related to the success of pregnancy [1]. Trophoblasts differentiate into various cell types that play a major role in implantation and placental development [2,3], as well as excreting essential

Received: Nov 5, 2017· Revised: Nov 29, 2017· Accepted: Jan 23, 2018 Corresponding author: Gi Jin Kim

Department of Biomedical Science, CHA University, 335 Pangyo-ro, Bundanggu, Seongnam 13488, Korea

Tel: +82-31-881-3687 Fax: +82-31-881-4102 E-mail: gjkim@cha.ac.kr

*This work was supported by the Basic Science Research Program through the National Research Foundation of Korea (NRF) funded by the Ministry of Science, ICT and Future Planning (NRF-2014R1A2A1A11050854).

This is an Open Access article distributed under the terms of the Creative Commons Attribution Non-Commercial License (http://creativecommons.org/licenses/by-nc/4.0/) which permits unrestricted non-commercial use, distribution, and reproduction in any medium, provided the original work is properly cited. substances during fetal development [4]. The important contribution of trophoblasts to a successful pregnancy has been established, whereas their role in implantation has not been researched in depth. Of particular note, the trophoblasts that lead to successful fetal development invade the endometrium and survive in hypoxic conditions [5]. Trophoblasts begin to invade the endometrium layer, and then fuse and accelerate villi formation, widening their surface area in order to make connections with maternal blood vessels. Trophoblasts thrive under hypoxic conditions until making contact with the maternal blood vessels.

Hypoxia, which is one of the several environmental factors that affect the implantation process, triggers changes in the expression of genes, enabling trophoblasts to adapt to their environment and carry out their various dynamic functions, such as proliferation, differentiation, and migration. However, severe hypoxia is regarded as dangerous and can lead to cell death. When a trophoblast contacts a 
maternal blood vessel, blood flows into the trophoblast. The sudden intervillous space perfusion increases the level of oxidative stress and triggers heat shock protein expression [6]. Thus, a high level of oxidative stress may contribute to the retardation of fetal development or even pregnancy failure [7]. As a process regulating oxidative stress, antioxidant genes such as heme oxygenases (HO-1 and $\mathrm{HO}-2)[8,9]$ and superoxide dismutase (SOD) are upregulated to reduce oxidative stress and to increase mitochondrial activity $[10,11]$. Failure of this regulatory process may be a factor leading to pregnancy failure. Thus, antioxidant supplementation in trophoblasts by injecting external antioxidant substances may increase the survival rate, invasive ability, and the mitochondrial activity of these cells.

Several previous studies have mentioned selenium as an antioxidant relevant to human health that protects many types of cells in the body from oxidative stress [12]. Recently, Khera and colleagues reported that selenium supplementation induced mitochondrial biogenesis in trophoblastic cancer cell lines (Swan-71, JEG-3, and BeWo) through the upregulated expression of the selenoprotein $\mathrm{H}, \mathrm{PGC}-1 \mathrm{a}$, and NRF-1 genes [13] and protected trophoblast cells from mitochondrial oxidative stress [14]. However, little is known about the effects of selenium on the migration ability of trophoblasts via mitochondrial oxidative stress and their relationships with other relevant factors.

Thus, we investigated whether selenium treatment affected the viability of trophoblasts and their migration activity, and whether it affected how the migration of trophoblasts is controlled via the regulation of mitochondrial oxidative stress.

\section{Methods}

\section{Cell culture}

Cells from the human extravillous trophoblast cell line HTR-8/Svneo were cultured under eight different conditions according to oxygen concentration, incubation time, and selenium treatment. The optimal concentration of selenium (Sigma-Aldrich, St. Louis, MO, USA) in the cells was determined by MTT (3-[4,5-dimethylthiazol-2-yl]-2,5 diphenyl tetrazolium bromide) assays. Cells $\left(2 \times 10^{3}\right)$ were plated into a 96-well cell culture plate and supplemented with selenium (with concentrations ranging from 0 to $12.5 \mathrm{nM}$ ) and cultured for 24 hours in normoxic and hypoxic conditions.

\section{Analysis of the cell survival rate}

To analyze the cell survival rate under conditions of selenium treatment and hypoxia, the trophoblast cells were plated into a 6-well plate $\left(6 \times 10^{4}\right.$ cells/well) and treated with $0.5 \mathrm{nM}$ selenium. After being cultured for 24 hours or 72 hours under normoxic or hypoxic conditions, the cells were stained with trypan blue and the number of cells was counted.

\section{Invasion assay}

Invasion assays were performed by using transwell plates containing polycarbonate filters with a pore size of $8.0 \mu \mathrm{m}$. The transwell inserts were first coated with $50 \mu \mathrm{L}$ of $1 \mathrm{mg} / \mathrm{mL}$ of Matrigel matrix (Becton Dickinson, Franklin Lakes, NJ, USA) at $37^{\circ} \mathrm{C}$ for 4 hours to allow gelling according to the manufacturer's recommendations. HTR8/SVneo cells were seeded at a density of $1.5 \times 10^{5}$ cells in $200 \mu \mathrm{L}$ of medium without fetal bovine serum in the upper chamber. The inserts were removed and washed in phosphate-buffered saline (PBS), and the nonmigrating cells in the upper chamber were removed with a cotton bud. The inserts were then fixed in cold methanol for 10 minutes at room temperature and stained with hematoxylin. Cells that invaded the lower surface were counted in 10 fields at $\times 200$ magnification.

\section{RNA extraction and reverse-transcription polymerase chain reaction}

Total RNA was extracted from the cultured cells using the Qiagen RNeasy kit. Reverse transcription was performed with $1 \mu \mathrm{g}$ of total RNA and Superscript III reverse transcriptase. The cDNA sequences were amplified by polymerase chain reaction (PCR). Real-time PCR for HO-1, HO-2, SOD, adenosine triphosphate (ATP) binding cassette subfamily $B$ member 10 (ABCB10), and hypoxia inducible factor 1a (HIF-1a) cDNA was performed using SYBR EX Taq (Roche, Basel, Switzerland) and Exicycler 96 quantitative thermal block (Bioneer, Daejeon, Korea). The PCR reaction conditions were as follows: denaturation at $95^{\circ} \mathrm{C}$ for 5 minutes, 40 cycles of $95^{\circ} \mathrm{C}$ for 20 seconds, an annealing temperature of $60^{\circ} \mathrm{C}$ for 15 minutes, extension at $70^{\circ} \mathrm{C}$ for 15 minutes, and a final extension at $72^{\circ} \mathrm{C}$ for 7 minutes. $\beta$-actin was used as an internal control.

\section{ATP assay}

ATP levels were measured using an ATP assay kit (Abcam, Cambridge, MA, USA) according to the manufacturer's instructions. Briefly, HTR-8/SVneo cells $\left(6 \times 10^{4}\right.$ cells/well) were seeded into a 6-well culture plate for 24 hours. The sample was the lysate from harvested HTR-8/SVneo cells and was quantified using the BCA assay (Pierce, Rockford, IL, USA). Aliquots of $10 \mu \mathrm{g}$ of lysate were added, amounting to no more than $10 \%$ of the total assay volume. The samples were added to a 96-well white plate and measured by the maximum emitted 560-nm fluorescence intensity using a Centro XS3 LB960 (Berthold technology, Oak Ridge, TN, USA).

\section{Measurement of reactive oxygen species and the membrane potential of mitochondria}

Levels of reactive oxygen species (ROS) and the membrane potential of mitochondria in HTR-8/SVneo trophoblast cells were deter- 
mined by MitoSOX/MitoTracker and JC-1 (Invitrogen, Camarillo, CA, USA) fluorescence staining methods, respectively. Cultured cells were seeded onto glass cover slips and washed with PBS. The cells were incubated with MitoSOX/MitoTracker at $37^{\circ} \mathrm{C}$. The cells were then washed with PBS and incubated with $1 \mu \mathrm{g} / \mathrm{mL}$ diamidino-phenylindole hydrochloride (Sigma-Aldrich) for 1 minute at room temperature. To evaluate the mitochondrial membrane potential, cells were incubated with $\mathrm{JC}-1$ for 20 minutes at $37^{\circ} \mathrm{C}$. The cells were mounted with mounting medium (Dako, Carpinteria, CA, USA) and the images were observed using a confocal laser scanning microscope imaging system.

\section{Statistical analysis}

All experiments were performed at least three times. Data are expressed as mean \pm standard error of the mean. Statistical significance between the control group and the selenium treatment group was determined using the Student $t$-test. For all analyses, $p$-values $<0.05$ were considered to indicate statistical significance.

\section{Results}

\section{Effects of selenium on trophoblast proliferation}

The optimal selenium concentration for trophoblasts was determined using MTT assays. In normoxic conditions, the proliferative activity of trophoblast cells was significantly higher at a selenium concentration of $5 \mathrm{nM}$, whereas proliferative activity under hypoxic conditions was maximized at a selenium concentration of $0.5 \mathrm{nM}(p<0.05)$
(Figure 1A). Based on these data, the viability of trophoblasts according to exposure to hypoxia and selenium was analyzed. No significant difference was found in the viability of trophoblasts in normoxic conditions regardless of selenium treatment; however, when exposed to selenium treatment under hypoxic conditions, their viability increased at 24 and 72 hours, although this trend did not reach statistical significance (Figure 1B). As shown in Figure $1 C$, the cell death rate of trophoblasts was increased by hypoxia; otherwise, selenium treatment significantly decreased the cell death rate. These data mean that selenium treatment affected the viability of trophoblasts under hypoxic conditions.

\section{Invasive ability of trophoblast cells under hypoxic conditions and selenium treatment}

It is well known that migration activity is a very important factor contributing to the implantation of trophoblasts into the maternal myometrium layer, and that hypoxia induces migration activity in trophoblasts through the activation of several signal molecules and adhesion molecules [15-17]. Therefore, we investigated whether selenium could control the migration of trophoblast cells. Trophoblast cells showed an increased invasive ability at 72 hours in both normoxic and hypoxic conditions. Interestingly, selenium treatment induced an increase in migration activity in all normoxic and hypoxic groups (Figure 2). These data suggest that selenium treatment enhances the migration activity of trophoblasts.

C

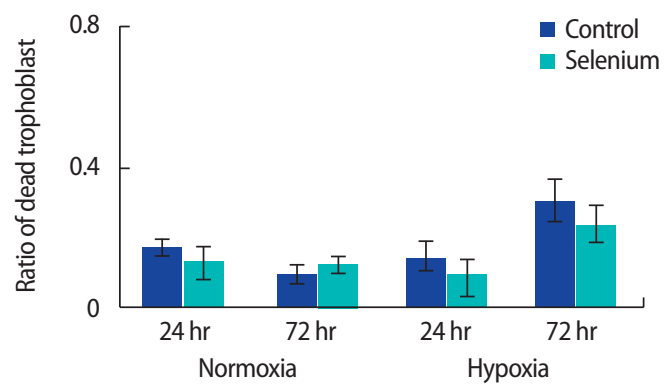

Figure 1. Selenium treatment increased the survival rate of trophoblasts under normoxic and hypoxic conditions. (A) MTT assay for selenium treatment in trophoblasts. To measure the effects of selenium in trophoblasts, trophoblasts in normoxic and hypoxic conditions were treated with various selenium concentrations ( ${ }^{*} p<0.05$ ). (B) The survival and $(\mathrm{C})$ death ratio of trophoblasts was determined by trypan blue staining under mormoxic and hypoxic conditions. The data were representative of three independent experiments and expressed as mean \pm standard error. MTT, 3-[4,5-dimethylthiazol-2-yl]-2,5 diphenyl tetrazolium bromide. 
(A)

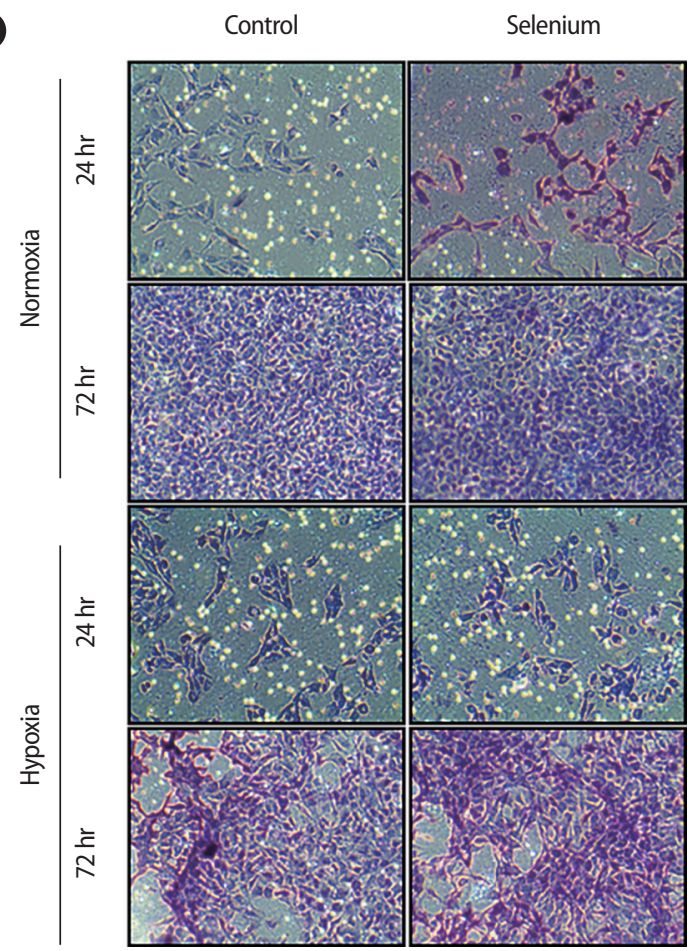

B

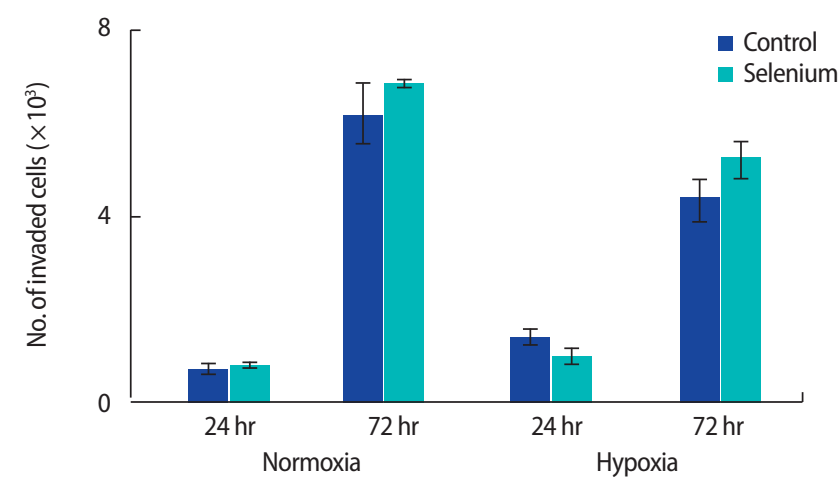

Figure 2. Selenium treatment increased the invasive ability of trophoblasts under normoxic and hypoxic conditions. The effects of selenium treatment on the invasive ability of trophoblasts were determined by $(A)$ an invasion assay based on hematoxylin staining (magnification, $\times 200$ ) and (B) invaded cell counting under normoxia and hypoxia conditions. The data were representative of three independent experiments and expressed as mean \pm standard error.

A

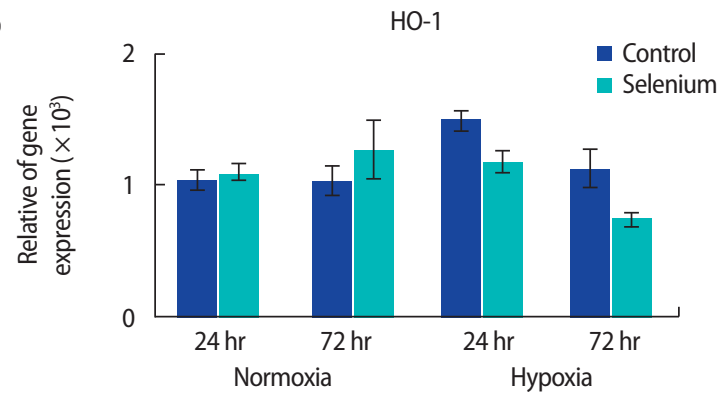

C

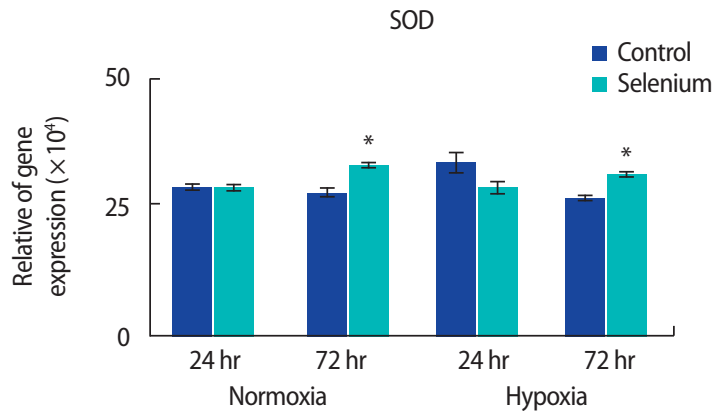

B

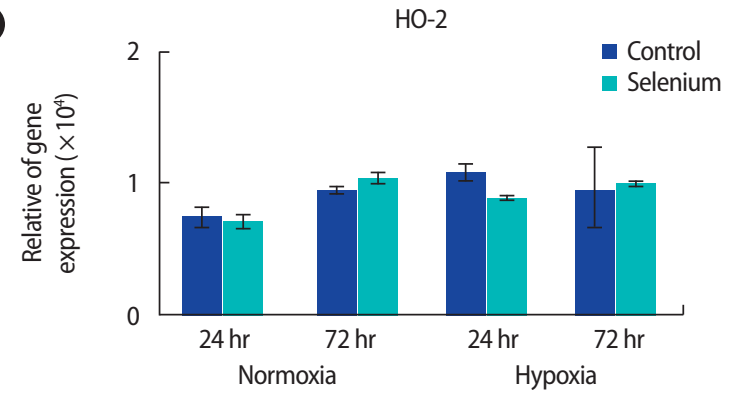

D

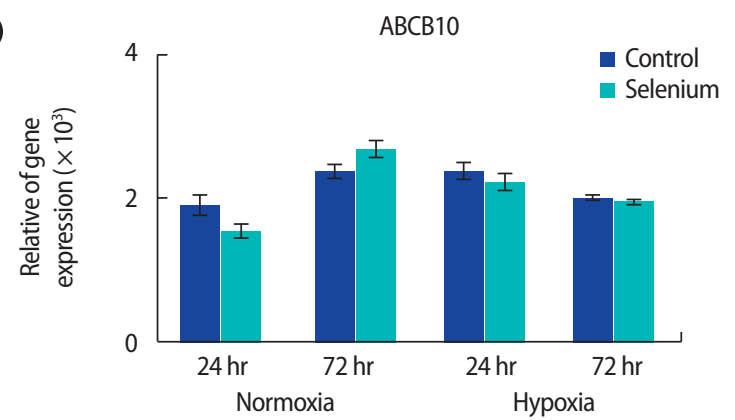

Figure 3. Selenium treatment changed the expression levels of antioxidant genes under normoxic and hypoxic conditions. (A) HO-1, (B) HO-2, (C) SOD, and (D) ABCB10 mRNA expression in trophoblasts according to treatment with selenium was determined by using qRT-PCR under normoxic and hypoxic conditions $\left({ }^{*} p<0.05\right)$. The data were representative of three independent experiments and expressed as mean \pm standard errors. $\mathrm{HO}$, heme oxygenase; SOD, superoxide dismutase; $A B C B 10$, adenosine triphosphate binding cassette subfamily $B$ member 10; qRT-PCR, quantitative real-time polymerase chain reaction. 


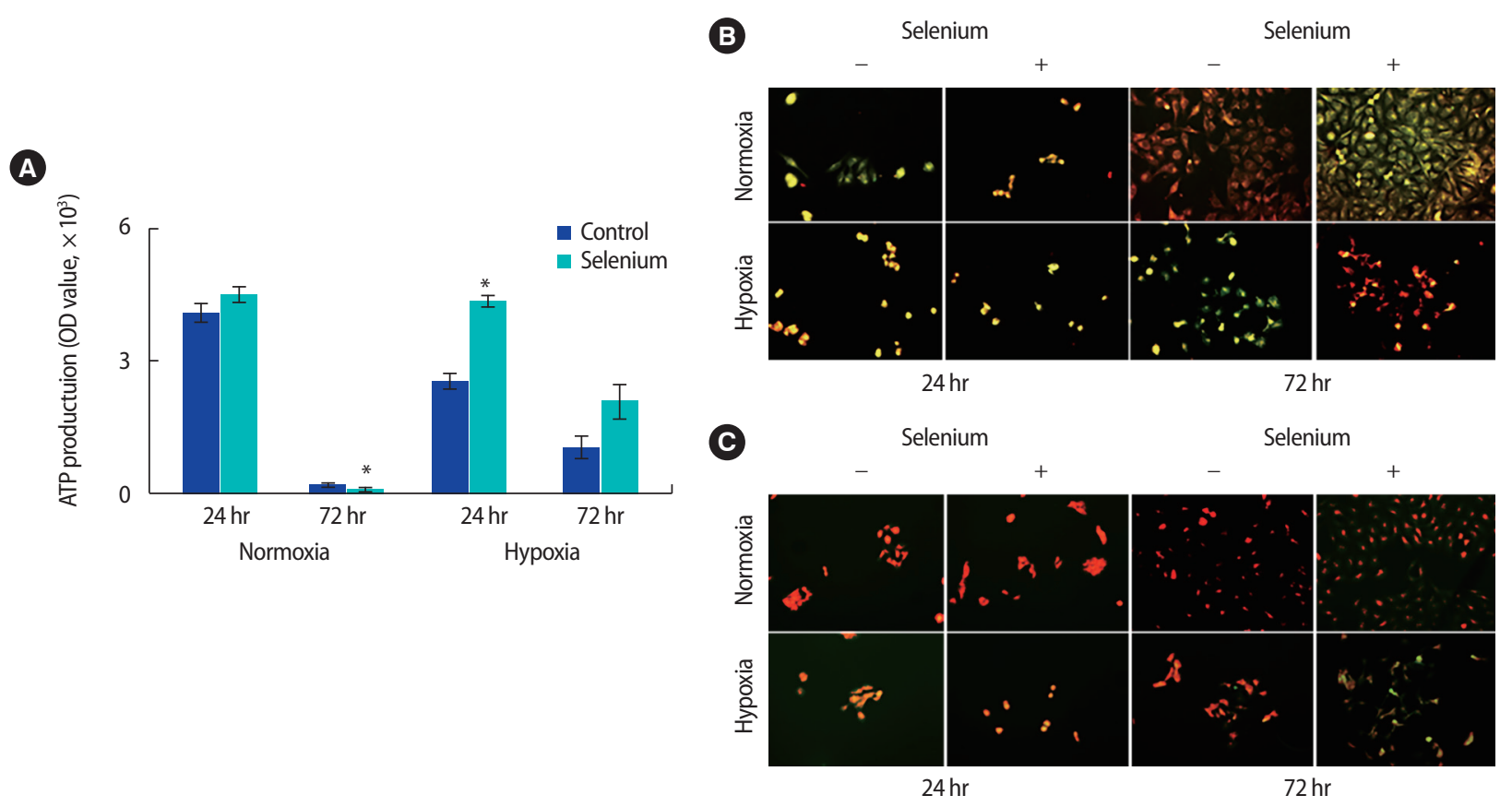

Figure 4. Effects of selenium on the mitochondrial function of trophoblasts under normoxic and hypoxic conditions. (A) The adenosine triphosphate production of trophoblasts was analyzed by a luminal fluorescence enzyme-linked immunosorbent assay under normoxic and hypoxic conditions $\left({ }^{*} p<0.05\right)$. (B) The mitochondrial membrane potential of trophoblast was analyzed by JC-1 fluorescent staining (magnification, $\times 200$ ). (C) Fluorescence images showed mitochondrial superoxide (MitoSOX Red) and mitochondrial content (MitoTracker Green) in trophoblasts according to selenium treatment under normoxic and hypoxic conditions (magnification, $\times 200$ ). The data were representative of three independent experiments and expressed as mean \pm standard error. OD, optical density.

\section{Effect of selenium on antioxidant enzyme expression in trophoblast cells under hypoxic conditions}

Based on these results, we investigated whether selenium reduced oxidative stress in the mitochondria of trophoblast cells. The expression of antioxidant enzyme genes according to selenium treatment was analyzed by real-time PCR (Figure 3). In normoxic conditions, HO-1 mRNA expression increased in trophoblast cells treated with selenium at 24 hours and 72 hours, while HO-1 expression gradually decreased in trophoblast cells exposed to hypoxia (Figure 3A). Additionally, $\mathrm{HO}-2$ expression in trophoblasts under normoxic conditions was increased by selenium, but there were no significant differences when trophoblast cells exposed to hypoxia were treated with selenium (Figure 3B). The expression of the ABCB10 gene, which contributes to heme biosynthesis and antioxidant regulation [18], increased under normoxic and hypoxic culture conditions for 24 hours. However, the expression of this gene was not significant after 72 hours of culture in either normoxic or hypoxic conditions (Figure 3D). Interestingly, SOD gene expression significantly increased in all groups treated with selenium at 72 hours $(p<0.05)$ (Figure $3 C$ ). These results suggest that selenium may affect oxidative stress in the mitochondria of trophoblasts depending on the oxygen concentration.

\section{Effects of selenium on mitochondrial function in trophoblasts}

Because we found that selenium affected oxidative stress in the mitochondria of trophoblasts, we analyzed the effects of selenium treatment on ATP production in trophoblasts. Generally, mitochondrial ATP production in trophoblasts decreased with long-term cultivation. In normoxic conditions, ATP production dramatically decreased at 72 hours compared to the levels observed at 24 hours. However, ATP production was significantly higher in all selenium-treated groups than in the selenium-untreated groups $(p<0.05)$ (Figure 4A). To evaluate the mitochondrial membrane potential and ROS levels of trophoblasts depending on selenium treatment under normoxic and hypoxic conditions, trophoblasts were analyzed using JC-1 and MitoSOX/Mitotracker, respectively.

In the JC-1 analysis, red indicates high mitochondrial membrane potential and green indicates the opposite. The mitochondrial membrane potential was higher in all selenium-treated groups, with a particularly dramatic increase noted in trophoblasts exposed to hypoxic conditions for 72 hours (Figure 4B). In the ROS analysis using MitoSOX/Mitotracker, red indicates high levels of ROS and green indicates low levels of ROS. In trophoblasts treated with selenium under hypoxic conditions for 72 hours, the ROS level was significantly lower (Figure 4C). These results suggest that selenium modulates the 
expression of antioxidant factors, thereby reducing the oxidative stress in the mitochondria of trophoblasts, thus increasing ATP production as well as the mitochondrial membrane potential in trophoblasts under hypoxic culture conditions.

\section{Discussion}

During the implantation process, trophoblasts are exposed to dynamic oxygen concentrations in the maternal myometrium. Because trophoblasts are implanted into the maternal myometrium layer 2 weeks after fertilization, trophoblasts are generally exposed to hypoxic conditions at the beginning of pregnancy and experience greater partial pressures of oxygen during normal placental development. However, if trophoblasts are persistently exposed to hypoxic conditions, they are known to cause a variety of gynecological diseases, such as preeclampsia and fetal growth retardation [19,20]. Thus, trophoblasts possess an innate ability to handle excessive oxidative stress through compensatory factors such as antioxidant enzymes.

In the present study, selenium-treated trophoblasts under hypoxic conditions showed increased viability, migration activity, and markers of mitochondrial functional activity, such as ATP production and membrane potential, resulting from downregulated ROS levels. A previous report showed that selenium acted as an anticancer drug that decreased the migration activity of murine melanoma cells via down-modulation of interleukin-18 expression, as well as inhibiting the proliferation and migration of HeLa cells $[21,22]$. However, our data showed that selenium induced a marked increase in the migration activity of trophoblasts, regardless of oxygen concentration. These results mean that selenium can contribute to a successful pregnancy by regulating the migration of trophoblasts. To our knowledge, this is the first report of the effects of selenium on enhancing migration and proliferation in trophoblasts exposed to hypoxic conditions through reducing mitochondrial oxidative stress.

Generally, antioxidant enzymes also contribute to oxidative stress regulation. Heme oxygenases, including $\mathrm{HO}-1$ and $\mathrm{HO}-2$, are important in regulating oxidative stress, and their expression can be sensitive to concentrations of oxygen in our circulatory system. However, their expression patterns and regulations are dynamic because of the complexities of $\mathrm{HO}$ regulation by hypoxia. Newby et al. [23] reported that HO-1 expression in syncytiotrophoblasts exposed to hypoxic conditions decreased, but otherwise, HO-2 expression was not affected by hypoxia in either cytotrophoblasts or syncytiotrophoblasts. These data are similar to our findings that HO-1 and SOD expression increased in trophoblast cells exposed to hypoxic conditions for 72 hours when treated with selenium. Additionally, $\mathrm{HO}-2$ was not induced by hypoxia or selenium treatment.
Hypoxia is a microenvironmental factor that induces a variety of biological events in cells, leading to the expression of HIF-1a. HIF-1a is known to be very unstable, as shown by its short half-life, so its expression decreases in the long term after exposure to hypoxic conditions [24]. However, antioxidant activity remains increased after the decrease in HIF-1a expression. The increase in oxidative stress in trophoblasts caused by hypoxia and the possibility of repeated reoxygenation cause mitochondrial insufficiency and, consequently, abnormal functions in trophoblasts, leading to obstetrical diseases such as preeclampsia $[25,26]$. Therefore, many researchers are studying the factors that can reduce these forms of oxidative stress to maintain a healthy pregnancy and to protect the health of mothers and babies, but insufficient progress has been made.

Recently, Mehta et al. [27] reported that selenium protected neurons in cases of focal cerebral ischemic damage by reducing oxidative stress and restoring mitochondrial function via stimulating mitochondrial biogenesis. Selenium can be predicted to play the role of a cofactor for these antioxidant enzymes, contributing to the significant increase in antioxidant enzymes. This oxidative stress regulation also increased the mitochondrial membrane potential and decreased ROS levels in trophoblast cells. These data are similar to ours (Figure $4 B, C)$.

In conclusion, we confirmed that selenium enhanced migration and proliferation in trophoblasts by reducing mitochondrial oxidative stress. Therefore, these results will be used as basic data for understanding the mechanisms of how trophoblast cells respond to oxidative stress and how selenium promotes the upregulation of related genes and improves cell survival rate and their invasive ability through regulating mitochondrial activity. Furthermore, these results suggest that selenium may be used in reproductive medicine for purposes including infertility treatment.

\section{Conflict of interest}

No potential conflict of interest relevant to this article was reported.

\section{References}

1. Kim GJ. Role of trophoblast in implantation and placenta development. Korean J Reprod Med 2010;37:181-9.

2. Aplin JD. Implantation, trophoblast differentiation and haemochorial placentation: mechanistic evidence in vivo and in vitro. J Cell Sci 1991;99(Pt 4):681-92.

3. Carter AM, Enders AC, Pijnenborg R. The role of invasive trophoblast in implantation and placentation of primates. Philos Trans R Soc Lond B Biol Sci 2015;370:20140070.

4. Red-Horse K, Zhou Y, Genbacev O, Prakobphol A, Foulk R, Mc- 
Master M, et al. Trophoblast differentiation during embryo implantation and formation of the maternal-fetal interface. J Clin Invest 2004;114:744-54.

5. Staun-Ram E, Shalev E. Human trophoblast function during the implantation process. Reprod Biol Endocrinol 2005;3:56.

6. Huppertz B, Gauster M, Orendi K, Konig J, Moser G. Oxygen as modulator of trophoblast invasion. J Anat 2009;215:14-20.

7. Poston L, Igosheva N, Mistry HD, Seed PT, Shennan AH, Rana S, et al. Role of oxidative stress and antioxidant supplementation in pregnancy disorders. Am J Clin Nutr 2011;94(6 Suppl):1980S1985 S.

8. Poss KD, Tonegawa S. Reduced stress defense in heme oxygenase 1-deficient cells. Proc Natl Acad Sci U S A 1997;94(20):1092530.

9. Appleton SD, Marks GS, Nakatsu K, Brien JF, Smith GN, Graham $\mathrm{CH}$, et al. Effects of hypoxia on heme oxygenase expression in human chorionic villi explants and immortalized trophoblast cells. Am J Physiol Heart Circ Physiol 2003;284:H853-8.

10. Frendo JL, Therond P, Bird T, Massin N, Muller F, Guibourdenche J, et al. Overexpression of copper zinc superoxide dismutase impairs human trophoblast cell fusion and differentiation. Endocrinology 2001;142:3638-48.

11. Burton GJ, Yung HW, Murray AJ. Mitochondrial-endoplasmic reticulum interactions in the trophoblast: stress and senescence. Placenta 2017;52:146-55.

12. Tinggi U. Selenium: its role as antioxidant in human health. Environ Health Prev Med 2008;13:102-8.

13. Khera A, Dong LF, Holland O, Vanderlelie J, Pasdar EA, Neuzil J, et al. Selenium supplementation induces mitochondrial biogenesis in trophoblasts. Placenta 2015;36:863-9.

14. Khera A, Vanderlelie JJ, Perkins AV. Selenium supplementation protects trophoblast cells from mitochondrial oxidative stress. Placenta 2013;34:594-8.

15. Arimoto-Ishida E, Sakata M, Sawada K, Nakayama M, Nishimoto F, Mabuchi S, et al. Up-regulation of alpha5-integrin by E-cadherin loss in hypoxia and its key role in the migration of extravillous trophoblast cells during early implantation. Endocrinology 2009;150:4306-15.

16. Darashchonak N, Sarisin A, Kleppa MJ, Powers RW, von VersenHoynck F. Activation of adenosine A2B receptor impairs properties of trophoblast cells and involves mitogen-activated protein
(MAP) kinase signaling. Placenta 2014;35:763-71.

17. Tian $Q$, Xue Y, Zheng W, Sun R, Ji W, Wang X, et al. Overexpression of hypoxia-inducible factor 1a induces migration and invasion through Notch signaling. Int J Oncol 2015;47:728-38.

18. Schaedler TA, Faust B, Shintre CA, Carpenter EP, Srinivasan V, van Veen $\mathrm{HW}$, et al. Structures and functions of mitochondrial $A B C$ transporters. Biochem Soc Trans 2015;43:943-51.

19. Zhao Y, Zheng YF, Luo QQ, Yan T, Liu XX, Han L, et al. Edaravone inhibits hypoxia-induced trophoblast-soluble Fms-like tyrosine kinase 1 expression: a possible therapeutic approach to preeclampsia. Placenta 2014;35:476-82.

20. Dobierzewska A, Palominos M, Irarrazabal CE, Sanchez M, Lozano M, Perez-Sepulveda A, et al. NFAT5 is up-regulated by hypoxia: possible implications in preeclampsia and intrauterine growth restriction. Biol Reprod 2015;93:14.

21. Song H, Kim J, Lee HK, Park HJ, Nam J, Park GB, et al. Selenium inhibits migration of murine melanoma cells via down-modulation of IL-18 expression. Int Immunopharmacol 2011;11:220813.

22. Sun L, Zhang J, Yang Q, Si Y, Liu Y, Wang Q, et al. Synergistic effects of SAM and selenium compounds on proliferation, migration and adhesion of HeLa cells. Anticancer Res 2017;37:443341.

23. Newby D, Cousins F, Myatt L, Lyall F. Heme oxygenase expression in cultured human trophoblast cells during in vitro differentiation: effects of hypoxia. Placenta 2005;26:201-9.

24. Yamanaka-Tatematsu M, Nakashima A, Fujita N, Shima T, Yoshimori T, Saito S. Autophagy induced by HIF1a overexpression supports trophoblast invasion by supplying cellular energy. PLoS One 2013;8:e76605.

25. Hung TH, Burton GJ. Hypoxia and reoxygenation: a possible mechanism for placental oxidative stress in preeclampsia. Taiwan J Obstet Gynecol 2006;45:189-200.

26. Xie Y, Zhou S, Jiang Z, Dai J, Puscheck EE, Lee I, et al. Hypoxic stress induces, but cannot sustain trophoblast stem cell differentiation to labyrinthine placenta due to mitochondrial insufficiency. Stem Cell Res 2014;13(3 Pt A):478-91.

27. Mehta SL, Kumari S, Mendelev N, Li PA. Selenium preserves mitochondrial function, stimulates mitochondrial biogenesis, and reduces infarct volume after focal cerebral ischemia. BMC Neurosci 2012;13:79. 